Selenwasserstoffsdure.

Die leichte Vereinigung des Schwefels mit dem Wasserstoff unter dem Einflusse von Bimstein, liess voraussehen, dass sie eben so bei Selen und Wasserstoff stattinden würde. Nach dem nämlichen Verfabren, wie vorber, erbält man Selenwasserstollsāure, welche durch alle ibre Eigenschaften leicht erkennbar ist.

Diese Versuche beweisen auf synthetische Weise die $\mathrm{Zu}$ sammensetzung des Jodwasserstoffes, Bromwasserstoffes, Schwe felwasserstoffes und Selenwasserstoffes. Es gab zwar keinen Zweifel über die Natur der Elemente, welche diese Wasserstolfsäuren bilden, da die Analygse uns hinreichend darüber aufgeklārt hat; aber es ist klar, dass das Interesse der Wissenschalt wächst, wenn die Beweise vervielfältigt werden und die beiden Arten der Nachiveisung, die Analyse und die Synthese zul den nämlichen Resultaten führen.

Der Gebrauch der porösen Kürper bci chemischen Reactionen verdient der Aufmerksamkeit. Es liegt in diesen Kürpern eine starke Kraft, welche wenige Kosten verursacht, und gestattet eine mässige Wärme anzuwenden, so dass sie vielleicht künftig in der Industrie Anwendung finden konnte.

\title{
LXII.
}

\section{Ueber die Zusammensetzung der Gase, die sich aus den Coaköfen entwickeln.}

\author{
Von \\ Ebolmen").
}

(Annal. des Mines. 4. S. Tosm. $X I X, 134$.

Man pllegt bekanallich die Steinkohlen besonders auf zweierlei Weise zu rerkoaken, einmal nämlich in Meilern oder Haufen, zweitens in besondern Oefen. Die erstere Methode ist nur an

-) Eine larze Notiz über diese Arbeit wrde bereits Bd LII, 296 gegeben.

D. Red. 
Orten üblich, wo die Steinkoblen zu sehr geringen Preisen zu baben sind; sie erforilert Kohle in grossen Stücken, giebt nur leichte Coaks und hat beträchtliche Verluste im Gefolge.

Die Verkoakung in Oefen geht regelmåssiger und sparsamer von Statten als die in Meilern. Man bedient sich derselben in Seraing zur Darstellung der für den Hohofenbetrieb erforderlichen Coaks. Ich hatte im September 1848 Gelegenheit, diese Verkoblung zu verfolgen und habe die dabei entweichenden Gase aufgefangen, um durch ihre Analyse Autscliluss über den Process wăhrend der Operation zu erlangen. Es war in der That zu untersuchen, ob die zur Verkohlung dienende Wärme durch die Verbrennung der Destillationsprodukte oder durch die Verbrennung eines Theils der schon gebildeten Coaks erzengt werde, oder ob beide Wirkungen gleichzeitig auftreten; endlich ob Jer in den Oefen eintretende Sauerstoff sich in Kohlensãure oder in Kohlenoxyd umändert.

Die in Seraing angewendeten Oefen*) liefern ihr Gas durch einen Seitenabzug des Haupt-Schornsteins unter Dampfkessel, wo durch eingeführlen Luftstrom eine Verbrennung der Gase bewerkstelligt werden kann.

Die angewendeten Kohlen gehðren zur Klasse der fetten und harten, sie liefern schwach aufgeblähte Coaks, die aber für den Hohofenbetrieb vortremich sind. Sie bestehen durchschnittlich aus

$$
\begin{aligned}
& \left.\begin{array}{rl}
78,00 & \text { p. C. } \begin{array}{l}
\text { Kohlenstoff } \\
2,00
\end{array} \text { Asche }
\end{array}\right\} \text { als Coak } \\
& \text { 20,00 " lüchtige Bestandtheile }
\end{aligned}
$$

und älıneln denen von Rochebelle bei Alais eben so in ihrer Zusammensetzung, wie in der Menge Coaks, welche sie geben.

Der Betrieb der Coakofen in Seraing ist folgender: Man ladet in jeden Ofen auf einmal 3 Cub.-Meter Kohlengruss, welches man 30 gut als möglich über die Sohle in einer Schicht von ungefahr $0, \mathrm{~m} 33$ Dicke ausbreitet. Hierauf schliesst man den mittleren oder die beiden seillichen Schornsteine und die

*) Ihre Beschreibang habell wir hier übergangen, da dieselbe in dem bekannten Werke ron Valerius äber die Gewinnang des Gusseisens wurahinlich gegeben- and darch Zeiohnung erläntert ist.

D. Red. 
beiden Thüren, ohne letztere zù verschmieren, und die Verkohlung beginnt. Man kann drei Abschnitte derselben unterscheiden: in dem ersten - ungefāhr $3 / 4$ Stunden umfassend - findet bloss Entwickelung von Wasserdămpfen statt; der 2 weite dauert ungefähr 1/2 Stunden. Die Gase entzünden sich und brennen zum Theil mit rother sebr russender Flamme, die Schornsteine sind vőllig geöffnet, die Thüren verschlossen. Im dritten $A b-$ schnitte brennen die Gase sehr gut obne Rauch und mit weisser Flamme. Die Kohle erscheint auf eine Erstreckung von $0^{\mathrm{m}}, 8$ $-0^{\mathrm{m}}, 10$ von der Oberfläche nach Innen glühend; dann verschmiert man die Thüren und lässt nur eine kleine Spalte in dem obern Theile des Thonlutums offen. Der Schornstein bleibt ganz offen. Wenn die Flamme kleiner wird, verschliesst man nach und nach vollstāndig die kleinen Spalten und wenn gar keine Flamme mehr erscheint, wird der Schornstein geschlossen. Eine Verkoakung mit Einschluss des Ladens und Ziehens dauert 22 bis 24 Stunden.

Es ist sehr wichtig, den Eintritt der Luft in den Oren gehörig zu regeln. Man hat beobachtet, dass die sehr fetten Kohlen mebr Luftzutritt erfordern, als die von Seraing und dass man dann die Oeffnungen für den Lufnutritt vergrössern mūsse, sonst würde die Verkohlung zu langsam vor sich gehen. Strőmt zu viel Luft ein, so erhält man viel Verlust und wenig dichte Coaks. Eine sehr langsame Verkoakung, die statt 24 Stunden 48 dauern würde, liefert sehr harte und dichte Coaks (Valerius).

Der mittlere Ertrag an Coaks in Seraing ist 160 p. C. dem Volum und 67 p. C. dem Gewicht nach.

Ich babe die aus dem Schornstein der Coaksöfen entweichenden Gase in drei verschiedenen Abschnitten der Verkohlung berausgezogen. Ein rechtwinkliges Glasrohr reichte ungefähr $0^{m}, 25$ in den Schornstein, und stand durch ein langes Caoutchoucrohr mit dem Rohr, welches die Gase aufnehmen sollte, in Verbindung. Dieses bestand aus einem ausgeblasenen Theile und zwei an dessen beiden Enden angelōtheten engen Glasrơhren, und war an beiden Enden durch zwei Hăhne verschliessbar. Nachdem der Apparat mit Gas gefült war, wurden die beiden Rỏbren zugeschmolzen, um ers* kurz vor der Analyse geöfinet zu werden. Die Analysen sind in dem Eudiometer von Journ. fo prakt. Chemie LV. 5. 
Regnault und Rei set ausgeführt. Zuerst liess ich die $\ddot{\mathrm{C}}$ durch $\dot{K} \dot{H}$ absorbiren, dann verpuffe ich mit Sauerstor nach Hinzufügung eines kleinen Theils Knallgas. Hieraul warde die Kohlensäure durch Kali weggescham und schliesslich eine Verpufung mit Ueberschuss ron WasserstoIr vorgenommen, um den absorbirten Sauerstoff za ermitteln. Versuche mit Phosphor an jedem Gase zeigten, dass kein freier Sauerstoff rorhanden sei.

Die Resultate sind:

\begin{tabular}{|c|c|c|c|c|}
\hline & Nach 2 Standen. & Nach $71 / 2$ St. & Nach $14 \mathrm{St}$. & $\begin{array}{l}\text { 4. } \\
\text { Mittel. }\end{array}$ \\
\hline $\begin{array}{l}\ddot{C}_{\mathrm{C}_{2}} \\
\mathrm{H}_{4}\end{array}$ & $\begin{array}{r}10,13 \\
1,44 \\
6,28\end{array}$ & $\begin{array}{l}9,60 \\
1,66 \\
3,07\end{array}$ & $\begin{array}{r}13,06 \\
0,40 \\
1,10\end{array}$ & $\begin{array}{r}10,93 \\
1,17 \\
3,68\end{array}$ \\
\hline & a. $100^{77,98}$ & $\begin{array}{r}3,91 \\
81,16\end{array}$ & $\begin{array}{r}2,19 \\
83,25\end{array}$ & $\begin{array}{r}3,42 \\
80,80\end{array}$ \\
\hline \multicolumn{2}{|c|}{$\begin{array}{l}\text { Sauerstoff a. } 100 \\
\text { Yol.Stickstoff } 15,7\end{array}$} & 14,2 & 17,0 & 15,6 \\
\hline
\end{tabular}

1. Gas; zwei Stunden nach dem Anzünden an einem der Seitenschornsteine aufgefangen. Rauch schwarz und dick, Flamme röthlich in Zwischenriumen erscbeinend.

2. Gas; 71/2 Stunde nach der Ladung genommen, Flamme glünzend, noch etwas röthlich, kein Rauch mehr.

3. Gas; nach 14 Stunden Destillation entnommen, helle, wenig umfangreiche Flamme; die Verkohlung scheint ihrem Ende nabe zu sein.

Prüfen wir jetzt, ob wir aus den Resultaten der Analyse etwas über die Art erfaluren, wie die Verkohlung vor sich geht. Wir llaben angenommen, dass die Steinkohle aus

$$
\begin{aligned}
& 4,85 \mathrm{H} \\
& 89,27 \mathrm{C} \\
& 4,470 \text { und } \mathrm{N} \\
& 1,41 \text { Asche }
\end{aligned}
$$

bestahd. Die Verkoakung in Oefen giebt nur 67 p. ci. Ceaks. Die theils durch die Destillation, theils durch die Verbrennung entwichenen Stoffe enthalten also folgerde Bestandiheile

$$
\begin{aligned}
& 4,85 \mathrm{H} \\
& 23,68 \mathrm{C} \\
& 4,470 \text { und } \mathrm{N} .
\end{aligned}
$$

Demaach wăre das Verbảltrios ron $\mathrm{C}: \mathrm{H}=1: 0,205$ dem Gerrichte nach. Betrachten wir im Gegentheil die mittlere 
Zusammensetzung der Gase, so findet man das Verbältniss von $\mathrm{C}: \mathbf{B}=7,76: 6,02$ dem Volum und 1:0,064 dem Gewichte nach. Man kann also hieraus den Schluss machen: dass mehr. als $2 / 3$ des in der Kohle enthaltenen Wasserstoffs während der Verkohlung verbrannt sind.

Wir haben in der That bisher auf den Kohlenstoff und Wasserstoff der condensirbaren Destillationsproducte, z. B. des Theers, noch keine Rücksicht genommen. Aber die Menge dieser Producle ist von keiner Erheblichkeit wegen der hohen T'emperatur, welche fast während der ganzen Dauer der Verkohlung die einschliessenden Mauern besitzen. Nur im Beginn der Operation sieht man sie in merkbarer Menge entweichen. Gleichfalls der hoben Temperatur der Matuer verdankt man das unbedeutende Auftreten Jes Kohlenwasserstofrs im Verhältniss zum Wasserstoff und Kohlenoxyd.

Die Verbrennung eines grossen Theils des in der Koble enthaltenen Wasserstonfs ist nicht ninder erwiesen Jurch den Vergleich der Mlenge des in den Gasen enthaltenen Sauerstofis mit der in ihnen entbaltenen Quantitä Stickstoff. Die Menge des gebundenen Sauerstoffs beträgt 15,63 auf 100 Stickstofr, wäbrend sie 26,26 betragen sollte, wenn aller atmosphärischer Stickstoff sich in den permanenten Gasen wieder finden müsste. Die Differenz 10,63 zeigt den Sauerstoff an, welcher zur Verbrennung des Wasserstoffs gedient hat. Also $2 / 5$ des atmosphärischen Sauerstoffs sind im Ofen in Wasser umgewandelt. (Hierbei ist die geringe Menge des in Jer Kohle enthaltenen Sauerstoffs unberücksichtigt gelassen.)

Aus dem Vergleiche des Stickstolfs mit dem Kohlenstoff in den untersucbten Gasen kann man auch das Volum Luft ermitteln, welches zur Verkoblung nơthig ist. Das Verhältuiss zwischer der Volumen des $N$ und $C$ ist $=80,8: 7,76$, dem Gewichte nach $80,8: 6,65=12,1: 1$. Da die atmosphärische Lun 77 Gewichtsprocente Stickstoff enthält, so findet man das Gewichtsverhältniss der Luft $z \mathrm{u}$ den in den Gasen enthaltenen Kohlenstofgewicht $=15,8: 1$. Da nun die durch die Gase entführte Menge C 23,68 p. C. vom Gewicht der Kohle beträgt, so verhält sich das Gewicht der zur Verkoakung gebrauchten Luft zum Gewicht der Kohle $=3,75: 1$. Man hat also $2^{c .} \times, 88$ Luft für jedes Kilo Kohle gebraucht; bei einer Ver- 
koblungsdauer von 20 Stunden erbält demnach der Coakofen durchschnittich $6^{C}$. $\$, 60$ Luf in der Minute, d. h. ungefăhr $2 / 3$ von der Menge, welche ein Holzkohlen-Hohofen erhält, der in 24 Stunden 2000 Kilogr. Gusseisen liefert.

Nun künnen wir auch den Wärme-Werth der gasigen Destillationsproducte ermitteln. Die bei den Coaküfen verloren gehende Wärme besteht 1. aus der merkbaren Wārme, welche dic Gase aus dem Ofen mitbringen, 2. aus der, welche sie durch Verbindung mit neuen Mengen Sauersloff entwickeln kőnnten. Die letztere zu ermitteln ist leicht. Nach Dulong entwickelt jedes Liter $H$ und $\dot{C}$ 3,13 Wärmeeinheiten, wenn es sich mit $1 / 2$ Liter Sauerstoff verbindet. $\mathrm{C}_{2} \mathrm{H}_{4}$ entwickelt 9,587 Einheiten. Die Gase des Coakofens werden also im Mittel jür jedes Liter 0,334 Wärmeeinheiten entwickeln, indem sie 0,0589 Liter Sauerstoff absorbiren. Da die ganze während der Verkoakung zutretende Luftmenge 7922 Cub.-M. betrïgt und jedes Liter Gas 1,02 Liter Luft entspricht, so betright also die Gesammtmenge der Gase 7767 Cub.-M. und diese werden bei der Verbrennung 2594178 Wärmeeinhciten entwickeln. Diese Wärmeyuantität ist aber offenbar nur ein Bruchtheil der ganzen verlornen Würme. Denn die Kohle verliert bei der Verkoakung 33 p. C. ihres Gewichts, bestehend aus $5,85 \mathrm{H}, 23,6 \mathrm{C}, 4,47 \mathrm{o}$. Nehmen wir den Stickstoff zu 1,5 p. C. in der Kohle an, so beträgt der zur nüthigen Oxydation des II und $C$ erforderliche Saucrstofr 101,95 und nach Abzug von 2,97 in der' Kohle euthaltenen Sauerstoffs 98,98. Das Aequivalent an Kohle der 33 p. C. brennbarer Stoffe ist 37,12. Setzt man als Wärmeentwickelungsvermógen des reinen C 8080, so findet man wach dem Welter'schen Gesetz fûr die einem Kilogr. Steinkohle entsprechende Wärmemenge 3000 Einheiten, also für 2750 Kilogr. 8250000 Einheiten. Davon ist aber ein Theil abzuziehen, welcher zur Erhitzung des Coaks bis zu einer hinlänglich hohen Temperatur verwendet wurde. Nehmen wir als solche $1000^{\circ}$ an und die spec. Wärme der Coaks 0,2 (Regnault), so werden die 1842 Kilo ( 67 p. C. von 2750 Kilo) 368400 Einheiten brauchen, d. h. $4 \frac{1}{2}$ p. C. der Gesammtmenge, man behälı also 7881600 Einheiten übrig.

Man sieht also, dass zur möglichsten Benutzung der Wärmemenge man die Heizapparate an den Coaksofen so nahe als moglich anbringen muss, damit die $2 / 3$ merkbarer Wärme benutzt 
werden und die Temperatur der Gase noch hoch genug sei, um sie angemessen verbrennen zu künnen. In Seraing hat man sehr befriedigende Resultate erhalten, denn 8 Coaksőfen speisen einen Kessel für 80 Pferdekraft.

Die 7881600 Wärmeeinheiten, welche die in 24 Stunden aus den Destillationsproducten entwickelte Wärme darstellen, sind ungefälır gleich 985 Kilo guter Steinkohle oder gleich 41 Kilo für die Stunde, d. I. 4,1 Kilo für 1 Pferdekraft und 1 Stunde.

Direcle Versuche in Seraing erwiesen, dass man durch die verloren gehende Wărme ungefähr 146 Kilo Wasser in der Stunde bei der mittleren Tension von $2 \mathrm{Atm}$. 76 verdampfen konnte.

Da die aus dem Ofen entweichenden Gase einen Ueberschuss brennbarer Kürper und sehr hohe Temperatur besitzen, so lassen sie sich bei ihrem Austritt leicht durch eine angemessene Mlenge zugemischter Luft völlig verbrennen. In den gewŏhnlichen Heerden der Dampfkessel betrügt tie Menge der eintretenden Iuft oft das Doppelte und Dreifache von der zur Verbrennung des Brennstoffs nüthigen, denn die Gase enthalten oft doppelt so viel Sauerstoff als Kohlensäure, wie es Co mbes*) durch zahlreiche Analysen dargethan hat. Dieser grosse Ueberschuss an Luft nuss offenbar einen beträchtlichen Verlust am wahren Nutzen des Brennmaterials verursachen, weil er mit hoher Temperatur aus dem Schornstein entweicht. Es würde daher vortheilhafter sein, die Steinkohlen in einem geschlossenen Raume zu verbrennen, welcher zu einer liohen Temperatur erhitzt ist und die Verbrennungsproducte durch eine Reihe von Oeffnungen in die Heiz-Canäle des Damplkessels schickt. Wenn der Raum und Gewölbe einmal erhitzt sind, würde die ganze Verbrennungswärme durch die Gase fortgelührt, die nur wenig oder gar keinen freien Sauerstoff enthielten. Dabei würde man zu gleicher Zeit die Bildung von Rauch vermindern und selbst ganz verhüten kōnnen.

Vergleicht man die Verkoakung mit der Holzverkohlung in Meilern, so gescheben beide durch die Einführung einer begrenzten Menge Luft zu dem zu verkohlenden Brennstoff. In den Coaksöfen verbrennen die Destillationsproducte grossentheils durch den

') Arm. des Mines.4. Ser. Tom. XI. 
Sauerstoff der Luft und entwickeln so die zur Verkoblung ndthige Wärme. Bei der Holzverkohlung in Meilern ist es dagegen ein Theil der schon gebildeten Koble, welche verbiennt, und die Destillationsproducte des Holzes entweichen unveründert, wenn nicht ganz doch wenigstens zum grơssten Theil. *) Die Ursache davon ist leicht anzugeben. Die Coaks nämlich sind schworer verbrennlich als dio Kohlenwasserstoffe, die bei der Destillation der Steinkohle entweichen. Dagegen sind die Destillationsproducte des Holzes, die sehr mit Wasserdămp̣fen und Kohlensãure beladen sind, schwerer verbrennlich als die Holzkoble. Letztere brennt an der Luft schon bei einer Temperatur von 230 $240^{\circ}$, die Destillationsproducte aber verlangen fast Rothgluth, ehe sie sich mit Sauerstoff verbinden.

Aus den angeführten Thatsachen ergeben sich folgende Schlüsse :

1. Die aus den Coakofen entweichenden Gase enthalten einen Theil der brennbaren Stoffe, welcher vom Beginn bis Ende der Verkohlung fortdauernd abnimmt.

2. Mehr als $2 / 3$ des in der Steinkohle enthaltenen Wasserstoffs verbrennen wăhrend der Verkoakung. Gleichzeitig verbrennt ein Theil des Koblenstoffs und zwar eine eben so grosse Menge als die bei der Destillation in geschlossenen Gefāssen entweichenden flüchtigen Producte enthalten. $2 / 5$ des in den Ofen eintretenden atmosphärischen Sauerstoffs bilden Wasser, $8 / 5$ vereinigen sich mit Kohlenstoff.

3. Von der Wärme, welche die Gase der Coaksöfen enthalten, sind ungefähr $2 / 3$ im freien Zustande, und diese muss man so nahe als möglich bei den Oefen benulzen. Da die Gase zur Entzündung mit Luft eine ziemlich hohe Temperatur gebrauchen, so kann man sie nicht, wie die Gase anderer Hüttenöfen, weit fortführen und erst dann verbrennen.

4. Die zur Verkohlung der Steinkohle nöthige Wărme wird in den Oefen einerseits durch die Verbrennung eines Theils der Destillationsproducte erzeugh, andererseits durch die Verbrennung einer merklichen Menge Koble. Letzteres isł der Hauptfehler

-) Vergl Ann. des Mines 4. Sor. Tom. MI. 
der Coaksơfen und man muss darnach streben, in ihnen fast eben so viel Coaks zu erhalten, als die Steinkohle in geschlossenen Gefässen giebt. Dass man diess erreichen könne, lāsst die beträchtliche Menge Wärme, welche die aus den Oefen entweichenden Gase enthalten, vermuthen.

\section{LXIII.}

\section{Not i z e n.}

1) Analyse des Mineralioassers des Celestins in Vichy.

Von Lefort.

(Journ. de Pharm. et Chim. T. XXVI, 14.)

Zu Tage gekommen bei einer Bohrung nahe der alten Quelle des Celestins. Temperatur $25^{\circ}$ C. Spec. Gew. $=1,0068$. 1000 Grammen enthielten

an Gasen:

$$
\begin{aligned}
& \text { N } 0,12 \text { C. C. } \\
& 0 \text { 0,025, } \\
& \ddot{\mathrm{c}} 0,519 \text {, }
\end{aligned}
$$

an festen Bestandtheilen:

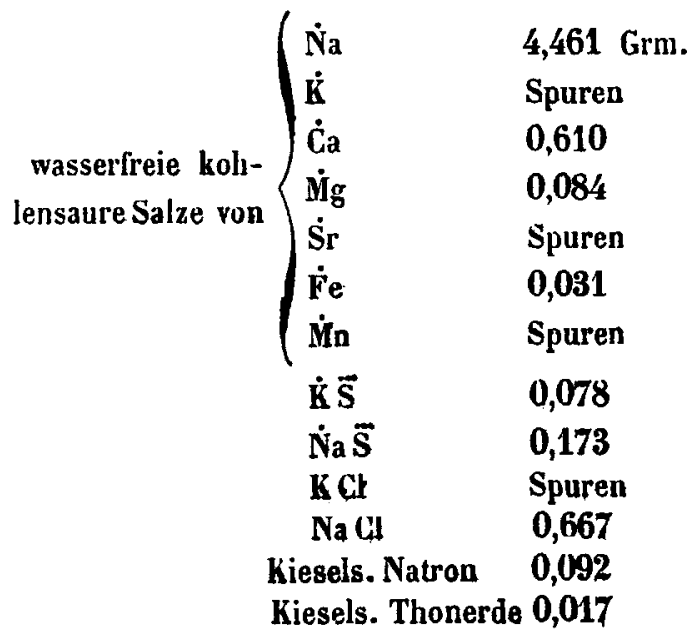

